\title{
SPECTRAL ORDER AUTOMORPHISMS ON HILBERT SPACE EFFECTS AND OBSERVABLES: THE 2-DIMENSIONAL CASE
}

\author{
LAJOS MOLNÁR AND GERGÖ NAGY
}

\begin{abstract}
In the earlier paper [6] we described the structure of all spectral order automorphisms of the sets of Hilbert space effects and bounded observables in the case where the dimension of the underlying Hilbert space is at least 3 . The aim of this note is to present a complete description in the missing two-dimensional case. We will see that in that case there is a one-to-one correspondence between the set of all spectral order automorphisms and the set of all bijective maps of pure states together with the set of all strictly increasing bijections of the real unit interval or the real line.
\end{abstract}

In von Neumann's mathematical formulation of quantum mechanics to every quantum system there corresponds a complex Hilbert space $H$ and the (bounded) observables are represented by (bounded) self-adjoint operators on $H$. We denote by $B_{s}(H)$ the space of all such operators and $\leq$ stands for the usual partial order on $B_{s}(H)$ (coming from the notion of positive semidefinitess). Although the order $\leq$ has apparent and important quantum mechanical meaning, as mentioned in the paper [6], it has certain disadvantages. In fact, Kadison [3] pointed out the surprising and strange property that the space $B_{s}(H)$ equipped with $\leq$ forms a so-called anti-lattice: the supremum of any two elements exists only in the trivial case where those elements are in fact comparable. This motivated Olson [7] to introduce another natural partial order $\preceq$ called spectral order on $B_{s}(H)$ with which this space becomes a conditionally complete lattice.

The definition of $\preceq$ is as follows. For any self-adjoint operator $A$ we denote by $E_{A}$ its spectral measure (defined on the Borel subsets of $\mathbb{R}$ ). For each pair $A, B \in B_{s}(H)$ we write

$$
A \preceq B \quad \text { if and only if } \quad E_{A}(-\infty, t] \geq E_{B}(-\infty, t], \quad t \in \mathbb{R} .
$$

Clearly, $\preceq$ is a partial order on $B_{s}(H)$. Moreover, one of the main results in [7] tells us that every nonempty subset of $B_{s}(H)$ which is bounded from above (with respect to this order) has a supremum and analogous statement holds true concerning the infimum. Beside the fact that the spectral order

2010 Mathematics Subject Classification. Primary: 47N50, 81R15. Secondary: 47B49. Key words and phrases. Spectral order, effects, observables.

The authors were supported by the "Lendület" Program (LP2012-46/2012) of the Hungarian Academy of Sciences. 
has nice mathematical properties, it has proved to be very useful in quantum theory, too. For a few of the latest references see [1] and [2] (Chapters 13 and 18).

In the paper [6] we considered the symmetries with respect to $\preceq$, i.e., the spectral order automorphisms (bijective maps which preserve the spectral order in both directions) of the sets of all (bounded) observables and all Hilbert space effects, respectively. We gave the complete descriptions of those transformations in the case where $\operatorname{dim} H \geq 3$ (in fact, as for observables, we also needed to assume that $\operatorname{dim} H<\infty$ ). The aim of this note is to complete our former results by presenting the structure of spectral order automorphisms in the missing case $\operatorname{dim} H=2$ which is rather important for different reasons, for example, for its use in quantum information science, see, e.g., [8]. We will show below that the structures of those automorphisms in the case $\operatorname{dim} H=2$ is rather different from the ones in the case $\operatorname{dim} H \geq 3$. This is rather surprising especially because there are no such differences in the case of the corresponding automorphims relative to the usual order $\leq$, see [4] (or Section 2.5 in [5]) and [9].

For the formulation of our results we need some terminology and notation. An operator $A \in B_{s}(H)$ is called an effect if $0 \leq A \leq I$ and the set of all such operators is denoted by $\mathcal{E}(H)$. Effects play important role in the quantum theory of measurements, they represent yes-no measurements which can be unsharp. In what follows $P_{1}(H)$ stands for the collection of all rank-one projections on $H$, its elements represent the pure states of the quantum system. Below we characterize the spectral order automorphisms of $\mathcal{E}(H)$ and $B_{s}(H)$ under the condition $\operatorname{dim} H=2$ which are the bijective maps $\phi$ of $\mathcal{E}(H)$ or $B_{s}(H)$ with the property that for any elements $A, B$ in their domains we have $\phi(A) \preceq \phi(B)$ if and only if $A \preceq B$.

The main results of the paper tell us that in the case $\operatorname{dim} H=2$ the spectral order automorphisms of $\mathcal{E}(H)$ and $B_{s}(H)$ originate from bijective maps (of any sort) of the set of all pure states together with strictly increasing bijections of the real interval $[0,1]$ and the real line $\mathbb{R}$, respectively. The precise statements read as follows.

Theorem 1. Suppose that $H$ is 2-dimensional and let $\phi: \mathcal{E}(H) \rightarrow \mathcal{E}(H)$ be a bijective map. Then $\phi$ is a spectral order automorphism if and only if there are bijections $\psi: P_{1}(H) \rightarrow P_{1}(H), f:[0,1] \rightarrow[0,1], f$ is strictly increasing and $\phi$ can be written in the form

$$
\phi(\lambda P+\mu Q)=(f(\mu)-f(\lambda)) \psi(Q)+f(\lambda) I,
$$

where $P, Q \in P_{1}(H)$ are arbitrary mutually orthogonal projections and $0 \leq$ $\lambda \leq \mu \leq 1$ are arbitrary numbers.

The characterization of spectral order automorphisms of $B_{s}(H)$ is similar.

Theorem 2. Suppose that $H$ is 2-dimensional and let $\phi: B_{s}(H) \rightarrow B_{s}(H)$ be a bijective map. Then $\phi$ is a spectral order automorphism if and only if 
there are bijections $\psi: P_{1}(H) \rightarrow P_{1}(H), f: \mathbb{R} \rightarrow \mathbb{R}, f$ is strictly increasing and $\phi$ can be written in the form

$$
\phi(\lambda P+\mu Q)=(f(\mu)-f(\lambda)) \psi(Q)+f(\lambda) I,
$$

where $P, Q \in P_{1}(H)$ are arbitrary mutually orthogonal projections and $\lambda \leq \mu$ are arbitrary real numbers.

In the proof of the above theorems we will use the following lemmas. The verification of the first one requires only elementary considerations, hence we omit the details. Recall that, by spectral theorem, if $H$ is 2-dimensional, then the elements of $B_{s}(H)$ are exactly the operators of the form $\lambda P+\mu Q$ where $P, Q \in P_{1}(H)$ are mutually orthogonal and $\lambda, \mu \in \mathbb{R}$ are such that $\lambda \leq \mu$, moreover $\lambda P+\mu Q$ is an effect exactly when $\lambda, \mu \in[0,1]$.

Lemma 1. Assume that $\operatorname{dim} H=2$ and let $A, B \in B_{s}(H)$. Let $P_{1}, Q_{1}$, respectively $P_{2}, Q_{2}$ be mutually orthogonal rank-one projections on $H$ and $\lambda_{1} \leq \mu_{1}, \lambda_{2} \leq \mu_{2}$ be real numbers such that $A=\lambda_{1} P_{1}+\mu_{1} Q_{1}$ and $B=$ $\lambda_{2} P_{2}+\mu_{2} Q_{2}$. We have $A \preceq B$ if and only if $\mu_{1} \leq \lambda_{2}$ or $P_{1}=P_{2}, \lambda_{1} \leq$ $\lambda_{2}, \mu_{1} \leq \mu_{2}$.

In the proof of the second lemma which gives a characterization of the scalar multiples of the identity in terms of the spectral order, we will use the following notation. For any $A, B \in B_{s}(H)$ with $A \preceq B$ we define

$$
[A, B]=\left\{X \in B_{s}(H) \mid A \preceq X \preceq B\right\} .
$$

We say that such a set is nondegenerate if its cardinality is at least 3 . For any $A \in B_{s}(H)$ let $\mathcal{M}_{A}$ be the set of those operators $X \in B_{s}(H)$ for which $A \neq X, A \preceq X,[A, X]$ is nondegenerate and there is an element $X^{\prime} \in B_{s}(H)$ such that $A \neq X^{\prime}, A \preceq X^{\prime},\left[A, X^{\prime}\right]$ is nondegenerate and $[A, X] \cap\left[A, X^{\prime}\right]=\{A\}$.

Lemma 2. Assume that $\operatorname{dim} H=2$ and let $A \in B_{s}(H)$. Then $A$ is a scalar multiple of the identity if and only if for all elements $X_{1}, X_{2} \in \mathcal{M}_{A}$ which are not comparable (with respect to $\preceq$ ) one has $\left[A, X_{1}\right] \cap\left[A, X_{2}\right]=\{A\}$.

Proof. First suppose that $A$ is a scalar operator. It is apparent that for any $B, C \in B_{s}(H)$ and $t \in \mathbb{R}$ we have $B \preceq C$ if and only if $B+t I \preceq C+t I$. Therefore, regarding the assertion in Lemma 2 , we may and do assume that $A=0$. Observe the following. For any $\lambda>0$ and $P \in P_{1}(H)$ we clearly have $0 \preceq \lambda P$ and one can check that

$$
[0, \lambda P]=\{\alpha P \mid \alpha \in[0, \lambda]\} .
$$

This immediately implies that if $P \neq Q \in P_{1}(H)$, then $[0, \lambda P] \cap[0, Q]=\{0\}$ which gives us that $\lambda P \in \mathcal{M}_{A}$. This shows that the positive scalar multiples of rank-one projections belong to the set $\mathcal{M}_{A}$. Conversely, it is easy to see that if $X \in B_{s}(H)$ is not a positive scalar multiple of an element in $P_{1}(H)$, then $X \notin \mathcal{M}_{A}$. It follows that $\mathcal{M}_{A}=\left\{\mu R \mid R \in P_{1}(H), \mu>0\right\}$. Now let $X_{1}, X_{2} \in \mathcal{M}_{A}$ be operators which are not comparable. Then we deduce 
that there are projections $P_{1}, P_{2} \in P_{1}(H)$ and numbers $\lambda_{1}, \lambda_{2}>0$ such that $X_{1}=\lambda_{1} P_{1}, X_{2}=\lambda_{2} P_{2}$. We infer that $P_{1} \neq P_{2}$ which, by (3), yields that $\left[0, X_{1}\right] \cap\left[0, X_{2}\right]=\{0\}=\{A\}$ and this completes the proof of the necessity part of the statement in Lemma 2.

For sufficiency, assume that $A$ is not a scalar operator. It is straightforward to check that beside addition by scalar multiples of the identity, multiplication by a fixed positive real number also respects the order $\preceq$. Hence without loss of generality we may and do suppose that $A \in P_{1}(H)$. Pick a projection $P \in P_{1}(H)$ such that $P, P^{\perp} \neq A$ and set $X_{1}=P+2 P^{\perp}, X^{\prime}=2 A$ $\left({ }^{\perp}\right.$ denotes the orthocomplementation on the set of all projections on $H$ ). Then one can check that $A \preceq X_{1}, X^{\prime}$ and $I \in\left[A, X_{1}\right]$, moreover we have $\left[A, X^{\prime}\right]=\{\lambda A \mid \lambda \in[1,2]\}$. Let $T \in\left[A, X_{1}\right] \cap\left[A, X^{\prime}\right]$. Then it follows that $T=\lambda_{0} A$ for some $\lambda_{0} \in[1,2]$. Since $T \preceq X_{1}$, referring to Lemma 1 we deduce that the eigenvalues of $T$ are at most 1 yielding that $T=A$. Therefore, we obtain that $X_{1} \in \mathcal{M}_{A}$. Let $Q \in P_{1}(H)$ be a projection such that $Q, Q^{\perp} \neq A, Q \neq P$ and set $X_{2}=Q+2 Q^{\perp}$. Just as above, we have $X_{2} \in \mathcal{M}_{A}$ and $I \in\left[A, X_{2}\right]$ which implies that $\left[A, X_{1}\right] \cap\left[A, X_{2}\right] \neq\{A\}$. Moreover, it is apparent that $X_{1}, X_{2}$ are not comparable. Putting together what we have proved above, we obtain the statement in Lemma 2.

We now turn to the proofs of our theorems.

Proof of Theorem 1. First, we assert that the bijections of $\mathcal{E}(H)$ of the form (1) are spectral order automorphisms. It is easy to see that for any stricly increasing bijection $f:[0,1] \rightarrow[0,1]$, the transformation $A \mapsto f(A), A \in$ $\mathcal{E}(H)$ is a spectral order automorphism of $\mathcal{E}(H)$. Therefore to prove the assertion we only have to verify the following claim. Given any bijection $\psi: P_{1}(H) \rightarrow P_{1}(H)$ and defining the transformation $\Psi: \mathcal{E}(H) \rightarrow \mathcal{E}(H)$ by the formula

$$
\Psi(\lambda P+\mu Q)=(\mu-\lambda) \psi(Q)+\lambda I=\lambda \psi(Q)^{\perp}+\mu \psi(Q),
$$

where $P, Q$ are mutually orthogonal rank-one projections on $H$ and $0 \leq \lambda \leq$ $\mu \leq 1$ are real numbers, we have that $\Psi$ is a spectral order automorphism of $\mathcal{E}(H)$. Easy application of Lemma 1 shows that this is really true.

Now, let $\phi$ be a spectral order automorphism of $\mathcal{E}(H)$. We recall that it follows from the results of Olson [7] that any nonempty subset $E \subset \mathcal{E}(H)$ has a supremum $\vee E$ and an infimum $\wedge E$ in $\mathcal{E}(H)$. In the next argument we borrow several steps and ideas from the proof of Theorem 3 in [6]. Just as there first we observe that $\wedge \mathcal{E}(H)=0$ which shows that $\phi(0)=0$. Next, one can prove that a nonzero effect $A$ on $H$ is of rank 1 if and only if it has the property that any two elements of $\mathcal{E}(H)$ preceding $A$ in the spectral order are comparable. Therefore $\phi$ preserves the rank-one elements of $\mathcal{E}(H)$ in both directions. Consequently, in our particular case where $\operatorname{dim} H=2$ we obtain that $\phi$ leaves the rank of effects invariant. The rank-one projections are precisely the maximal elements of the collection of all rank-one operators in $\mathcal{E}(H)$. This implies that $\phi$ preserves the rank-one projections on $H$ in 
both directions and therefore its restriction $\psi$ to $P_{1}(H)$ is a bijection of $P_{1}(H)$.

Let $P \in P_{1}(H)$ be a projection and $0 \leq \lambda \leq 1$ be a real number. It is clear that $\lambda P \preceq P$ and therefore $\phi(\lambda P) \preceq \phi(P)$ which, by (3), yields the existence of a number $f_{P}(\lambda)$ for which

$$
\phi(\lambda P)=f_{P}(\lambda) \phi(P) .
$$

Since $\phi$ is a spectral order automorphism, we see that $f_{P}$ is a strictly increasing bijection of the unit interval $[0,1]$. Next, following the arguments in the second and third paragraphs on p. 247 in [6], one can show that $f_{P}$ in fact does not depend on $P$, hence we may and do denote it simply by $f$. Clearly, the map $A \mapsto f^{-1}(A), A \in \mathcal{E}(H)$ is a spectral order automorphism of $\mathcal{E}(H)$. This gives us that the transformation $\Phi: \mathcal{E}(H) \rightarrow \mathcal{E}(H)$ defined by $\Phi(A)=f^{-1}(\phi(A)), A \in \mathcal{E}(H)$ is again a spectral order automorphism and, by (4), it has the additional property that for any projection $P \in P_{1}(H)$ and real number $0 \leq \lambda \leq 1$, the equality

$$
\Phi(\lambda P)=\lambda \Phi(P)
$$

holds true. Since $\phi$ and $\Phi$ coincide on the set of all projections, it is also clear that the restriction of $\Phi$ onto $P_{1}(H)$ is equal to $\psi$.

Now pick arbitrary rank-one projections $P, Q$ on $H$ and real numbers $0 \leq \lambda, \mu \leq 1$ such that $P \neq Q$ and $\lambda \leq \mu$. By the second paragraph on page 247 in [6] we have

$$
\vee\{\lambda P, \mu Q\}=\lambda(P \vee Q-Q)+\mu Q=\lambda Q^{\perp}+\mu Q .
$$

Assume that $P$ and $Q$ are orthogonal to each other. We then compute

$$
\begin{gathered}
\Phi(\lambda P+\mu Q)=\Phi(\vee\{\lambda P, \mu Q\})=\vee\{\Phi(\lambda P), \Phi(\mu Q)\}=\vee\{\lambda \Phi(P), \mu \Phi(Q)\} \\
=\lambda \Phi(Q)^{\perp}+\mu \Phi(Q)=\lambda \psi(Q)^{\perp}+\mu \psi(Q)=(\mu-\lambda) \psi(Q)+\lambda I .
\end{gathered}
$$

Having in mind the definition of $\Phi$ we obtain that the original transformation $\phi$ can be written in the form (1).

Keeping the assumption $\operatorname{dim} H=2$, for the proof of Theorem 2 we will need a characterization of those spectral order automorphisms of the set of all positive operators which leave the nonnegative scalar multiples of the identity fixed. We argue as follows.

For any bijection $\varphi: P_{1}(H) \rightarrow P_{1}(H)$ define

$$
\Theta_{\varphi}(\lambda P+\mu Q)=(\mu-\lambda) \varphi(Q)+\lambda I=\lambda \varphi(Q)^{\perp}+\mu \varphi(Q),
$$

whenever $P, Q$ are mutually orthogonal rank-one projections and $0 \leq \lambda \leq \mu$ are real numbers. It follows easily from Theorem 1 that the transformations $\left.\Theta_{\varphi}\right|_{\mathcal{E}(H)}$ are exactly the spectral order automorphisms of $\mathcal{E}(H)$ which fix the scalar elements of $\mathcal{E}(H)$. We now assert that the transformations $\Theta_{\varphi}$ defined in (5) are exactly the spectral order automorphisms of the set of positive operators which fix the nonnegative scalar multiples of the identity. By Lemma 1 we see that every $\Theta_{\varphi}$ is such a transformation. For the converse, let 
$\Theta$ be a bijection of the set of all positive elements of $B_{s}(H)$ which preserves the spectral order in both directions and which fixes the nonnegative scalar multiples of the identity. Since $\Theta$ leaves $\mathcal{E}(H)$ invariant, by Theorem 1 there is a bijection $\varphi: P_{1}(H) \rightarrow P_{1}(H)$ such that $\Theta(A)=\Theta_{\varphi}(A)$ holds for all $A \in \mathcal{E}(H)$. Let $c>1$ be any number and consider the map $A \mapsto$ $(1 / c) \Theta(c A), A \in \mathcal{E}(H)$. Clearly, this map is a spectral order automorphism of $\mathcal{E}(H)$ and hence we have a bijection $\varphi_{c}: P_{1}(H) \rightarrow P_{1}(H)$ such that $(1 / c) \Theta(c A)=\Theta_{\varphi_{c}}(A), A \in \mathcal{E}(H)$. If $c A \in \mathcal{E}(H)$, we deduce

$$
(1 / c) \Theta(c A)=(1 / c) \Theta_{\varphi}(c A)=\Theta_{\varphi}(A) .
$$

Consequently, whenever $c A \in \mathcal{E}(H)$, we have $\Theta_{\varphi_{c}}(A)=\Theta_{\varphi}(A)$. This easily implies that $\varphi_{c}=\varphi$ and thus we obtain that

$$
\Theta(c A)=c \Theta_{\varphi_{c}}(A)=c \Theta_{\varphi}(A)=\Theta_{\varphi}(c A)
$$

holds for any $A \in \mathcal{E}(H)$ and $c>1$. This yields $\Theta=\Theta_{\varphi}$ verifying our claim.

We are now in a position to prove our second theorem. The fundamental idea of the proof is similar to the one in the proof of Theorem 4 in [6] but the technical details are rather different.

Proof of Theorem 2. Let $\phi: B_{s}(H) \rightarrow B_{s}(H)$ be a spectral order automorphism. First, referring to Lemma 2 we deduce that $\phi$ preserves the scalar operators in both directions. This implies that there is a strictly increasing bijection $f: \mathbb{R} \rightarrow \mathbb{R}$ such that for all real numbers $\lambda$ we have $\phi(\lambda I)=f(\lambda) I$. Now consider the transformation $\Phi: B_{s}(H) \rightarrow B_{s}(H)$ defined by $\Phi(A)=f^{-1}(\phi(A)), A \in B_{s}(H)$. Clearly, $\Phi$ is a spectral order automorphism with the additional property that it leaves the scalar operators fixed. It follows that $\Phi$ maps $\mathcal{E}(H)$ onto itself and the restriction of $\Phi$ onto $\mathcal{E}(H)$ is a spectral order automorphism of $\mathcal{E}(H)$. Applying Theorem 1 we obtain that there exist a bijection $\psi: P_{1}(H) \rightarrow P_{1}(H)$ and a strictly increasing bijection $g:[0,1] \rightarrow[0,1]$ such that $\left.\Phi\right|_{\mathcal{E}(H)}$ is of the form

$$
\Phi(\lambda P+\mu Q)=(g(\mu)-g(\lambda)) \psi(Q)+g(\lambda) I,
$$

where $P, Q \in P_{1}(H)$ are arbitrary mutually orthogonal projections and $0 \leq$ $\lambda \leq \mu \leq 1$ are arbitrary numbers. In fact, since $\Phi$ leaves the scalar operators fixed, we infer that $g$ is the identity on $[0,1]$.

Now define a map $\Psi: B_{s}(H) \rightarrow B_{s}(H)$ by the formula

$$
\Psi(\lambda P+\mu Q)=(\mu-\lambda) \psi(Q)+\lambda I=\lambda \psi(Q)^{\perp}+\mu \psi(Q),
$$

where $P, Q$ are mutually orthogonal rank-one projections on $H$ and $\lambda \leq \mu$ are arbitrary real numbers. By Lemma $1, \Psi$ is a spectral order automorphism of $B_{s}(H)$. Furthermore, we see that $\Psi$ and $\Phi$ coincide on $\mathcal{E}(H)$ (and leave it invariant) and they both fix the scalar multiples of the identity. Consider $\Gamma=\Psi^{-1} \circ \Phi$. This is again a spectral order automorphism of $B_{s}(H)$ which fixes the scalar multiples of the identity and acts as the identity on $\mathcal{E}(H)$. We prove that it is the identity on the whole set $B_{s}(H)$. Considering $\Gamma$ on the set of positive elements of $B_{s}(H)$ we easily obtain from the discussion 
preceding the present proof that $\Gamma$ acts as the identity on the set of all positive elements of $B_{s}(H)$.

Let $B \in B_{s}(H)$ be arbitrary. There is a scalar $0>k \in \mathbb{R}$ such that $B-k I$ is positive. Consider the transformation

$$
C \longmapsto \Gamma(C+k I)-k I, \quad 0 \leq C \in B_{s}(H) .
$$

It is easy to see that this is a bijective map on the set of all positive elements of $B_{s}(H)$ which preserves the spectral order in both directions and sends every nonnegative scalar multiple of the identity to itself. We apply the discussion preceding the proof to see that there is a bijection $\varphi_{k}: P_{1}(H) \rightarrow$ $P_{1}(H)$ such that

$$
\Gamma(C+k I)-k I=\Theta_{\varphi_{k}}(C), \quad 0 \leq C \in B_{s}(H) .
$$

If $C \geq-k I$, then $C+k I \geq 0$ and, because $\Gamma$ acts as the identity on positive elements, we have

$$
C=(C+k I)-k I=\Gamma(C+k I)-k I=\Theta_{\varphi_{k}}(C) .
$$

It follows easily that $\varphi_{k}$ is the identity. Setting $C=B-k I$, we obtain

$$
\Gamma(B)-k I=\Gamma((B-k I)+k I)-k I=\Gamma(C+k I)-k I=\Theta_{\varphi_{k}}(C)=C
$$

and this implies $\Gamma(B)=C+k I=B$. Therefore, we obtain that $\Gamma$ equals the identity on the whole set $B_{s}(H)$. It follows that $\Phi=\Psi$ and having in mind the definition of $\Phi$ we conclude that $\phi$ is of the desired form (2). This proves the necessity part of the theorem. The sufficiency follows just as in the first part of the proof of Theorem 1 .

REMARK. We conclude the paper with some remarks. For any linear subspace $M$ of a finite dimensional complex Hilbert space $H$, let us denote by $P_{M}$ the orthogonal projection onto $M$. We recall the following statement which is a part of Proposition 1 in [6]:

Supposing that $H$ is finite dimensional and $T: H \rightarrow H$ is a bijective semilinear operator, for any self-adjoint operator $A$ on $H$ there exists a unique compactly supported spectral measure $E_{A}^{T}$ on the Borel subsets of $\mathbb{R}$ such that for any real number $t$ we have

$$
E_{A}^{T}(-\infty, t]=I-P_{T\left(\operatorname{rng} E_{A}(t, \infty)\right)} .
$$

Next, define the map $\psi_{T}: B_{s}(H) \rightarrow B_{s}(H)$ by

$$
\psi_{T}(A)=\int_{\mathbb{R}} \lambda d E_{A}^{T}(\lambda), \quad A \in B_{s}(H) .
$$

Theorem 4 in [6] tells us that in the case where $3 \leq \operatorname{dim} H<\infty$, the spectral order automorphisms of $B_{s}(H)$ are exactly the transformations of the form

$$
A \mapsto \psi_{T}(f(A)),
$$

where $T: H \rightarrow H$ is a bijective semilinear operator and $f$ is a strictly increasing bijection of $\mathbb{R}$. 
In fact, we learn from the arguments given in [6] that the maps in the last displayed formula are spectral order automorphisms also in the twodimensional case. Now, the question arises if there are spectral order automorphisms in that case which are not of that form. By the following example we show that if $\operatorname{dim} H=2$, the group of spectral order automorphisms of $B_{s}(H)$ is strictly larger than the collection of all maps of the form (7). To see this, we first recall that in [10, section 5.2] it was proved that in the case $\operatorname{dim} H=2$ there is an orthogonality preserving bijection $\psi$ of $P_{1}(H)$ which does not leave the transition probability invariant. Consider the transformation $\Psi$ corresponding to $\psi$ defined by the formula (6). We already know that $\Psi$ is a spectral order automorphism of $B_{s}(H)$.

We assert that $\Psi$ cannot be written in the form (7). Assume on the contrary that there exists a bijective semilinear operator $T: H \rightarrow H$ and a strictly increasing bijective map $f: \mathbb{R} \rightarrow \mathbb{R}$ such that $\Psi(A)=\psi_{T}(f(A)), A \in$ $B_{s}(H)$. There is a ring automorphism $h$ of $\mathbb{C}$ such that for any vector $x \in H$ and for each complex number $\lambda$ one has

$$
T(\lambda x)=h(\lambda) T x .
$$

It is easy to see that for all self-adjoint operators $A \in B_{s}(H)$ one has $\psi_{T}(f(A))=f\left(\psi_{T}(A)\right)$. Let $x \in H$ be a unit vector. We infer that for the operator $|x\rangle\langle x| \in P_{1}(H)$ the equality

$$
\psi(|x\rangle\langle x|)=\Psi(|x\rangle\langle x|)=f\left(\psi_{T}(|x\rangle\langle x|)\right)
$$

holds true. A straightforward calculation shows that $\psi_{T}(|x\rangle\langle x|)$ is the projection onto the subspace generated by the nonzero vector $T x$ which means that $\psi_{T}(|x\rangle\langle x|)=\|T x\|^{-2}|T x\rangle\langle T x|$. Since $f\left(\psi_{T}(|x\rangle\langle x|)\right)$ needs to be a projection, we deduce that $f\left(|| T x \|^{-2}|T x\rangle\langle T x|\right)=\|T x\|^{-2}|T x\rangle\langle T x|$ which yields the equality $\psi(|x\rangle\langle x|)=\|T x\|^{-2}|T x\rangle\langle T x|$.

Using the orthogonality preserving property of $\psi$, it follows that $T$ also has this property, i.e., for any vectors $x, y \in H$ we have $\langle T x, T y\rangle=0$ exactly when $\langle x, y\rangle=0$. Now choose two mutually orthogonal unit vectors $u, v$ from $H$ and a complex number $\lambda$. Then we clearly have $\langle\lambda u+v, u-\bar{\lambda} v\rangle=$ 0 and therefore $\langle h(\lambda) T u+T v, T u-h(\bar{\lambda}) T v\rangle=0$. Having in mind the equalities $h(1)=1$ and $\langle T u, T v\rangle=0$, we get that $\|T u\|=\|T v\|$ and then we obtain $h(\bar{\lambda})=\overline{h(\lambda)}$ which yields $h(\mathbb{R}) \subset \mathbb{R}$. Since the only nonzero field endomorphism of $\mathbb{R}$ is the identity, we deduce that $h$ is either the identity or the conjugation on $\mathbb{C}$ which implies that $T$ is either linear or conjugate linear. By the orthogonality preserving property of $T$, for any vectors $x, y \in H$ with $\langle x, y\rangle=0$ we have $\left\langle T^{*} T x, y\right\rangle=0$ yielding that $T^{*} T$ is a scalar operator. We then get that $T$ is a positive scalar multiple of a unitary or antiunitary operator $U$. Finally, we conclude that $\psi(P)=$ $U P U^{*}, P \in P_{1}(H)$ and thus $\psi$ preserves the transition probability which is a contradiction. Consequently, $\Psi$ cannot be written in the form (7).

One can verify in a similar manner that in the case where $\operatorname{dim} H=2$, the group of spectral order automorphisms of $\mathcal{E}(H)$ is also strictly larger than 
in higher dimensions. In fact, one needs to refer to Theorem 3 instead of Theorem 4 in [6] what we have used above.

\section{REFERENCES}

1. A. Döring and B. Dewitt, Self-adjoint operators as functions I: lattices, Galois connections, and the spectral order, Comm. Math. Phys. 328 (2014), 499-525.

2. C. Flori, A First Course in Topos Quantum Theory, Lecture Notes in Physics, vol. 868, Springer, 2013.

3. R.V. Kadison, Order properties of bounded self-adjoint operators, Proc. Amer. Math. Soc., 2 (1951), 505-510.

4. L. Molnár, Order-automorphisms of the set of bounded observables, J. Math. Phys. 42 (2001), 5904-5909.

5. L. Molnár, Selected Preserver Problems on Algebraic Structures of Linear Operators and on Function Spaces, Lecture Notes in Mathematics, Vol. 1895, Springer, Berlin Heidelberg, 2007.

6. L. Molnár and P. Šemrl, Spectral order automorphisms of the spaces of Hilbert space effects and observables, Lett. Math. Phys. 80 (2007), 239-255.

7. M. P. Olson, The selfadjoint operators of a von Neumann algebra form a conditionally complete lattice, Proc. Am. Math. Soc. 28 (1971), 537-544.

8. D. Petz, Quantum Information Theory and Quantum Statistics, Springer, BerlinHeidelberg, 2008.

9. P. Šemrl, Comparability preserving maps on Hilbert space effect algebras, Comm. Math. Phys. 313 (2012), 375-384.

10. U. Uhlhorn, Representation of symmetry transformations in quantum mechanics, Ark. Fysik 23 (1963), 307-340.

Department of Analysis, Bolyai Institute, University of Szeged, H-6720 Szeged, Aradi vértanúk tere 1., Hungary and MtA-DE "Lendület" Functional Analysis Research Group, Institute of Mathematics, University of Debrecen, H-4002 Debrecen, P.O. Box 400, Hungary

E-mail address: molnarl@math.u-szeged.hu

URL: http://www.math.u-szeged.hu/ molnarl/

Mta-DE "Lendület" Functional Analysis Research Group, Institute of Mathematics, University of Debrecen, H-4002 Debrecen, P.O. Box 400, HunGARY

E-mail address: nagyg@science.unideb.hu 